\title{
Soil organic carbon estimation using VIS-NIR-SWIR spectroscopy: The effect of multiple sensors and scanning conditions
}

\author{
Asa Gholizadeh ${ }^{1 *}$, Carsten Neumann ${ }^{2}$, Sabine Chabrillat ${ }^{2}$, Bas van Wesemael ${ }^{3}$, Fabio Castaldi ${ }^{4}$, Luboš Borůvka ${ }^{1}$, \\ Aleš Klement ${ }^{1}$, Christian Hohmann ${ }^{2}$ \\ ${ }^{I}$ Department of Soil Science and Soil Protection, Faculty of Agrobiology, Food and Natural Resources, Czech \\ University of Life Sciences Prague, Kamycka 129, 16500 Prague, Czech Republic \\ ${ }^{2}$ Helmholtz Center Potsdam GFZ German Research Centre for Geosciences, Section 1.4 Remote Sensing, \\ Telegrafenberg, 14473 Potsdam, Germany \\ ${ }^{3}$ Georges Lemaitre Centre for Earth and Climate Research, Earth and Life Institute, Universite Catholique de \\ Louvain, 1348 Louvain-la-Neuve, Belgium \\ ${ }^{4}$ ILVO_Flanders Research Institute for Agriculture, Fisheries and Food, Technology and Food Science-Agricultural \\ Engineering, 9820 Merelbeke, Belgium \\ *Corresponding author: gholizadeh@af.czu.cz
}

\begin{abstract}
Visible-near infrared-shortwave infrared (VIS-NIR-SWIR) spectroscopy is being increasingly used for soil organic carbon (SOC) assessment. Common practice consists of scanning soil samples using a single spectrometer. Considerations have rarely been documented of the effects of using multiple instruments and scanning conditions on SOC model calibration that occur when merging soil spectral libraries (SSLs), particularly in soils with low SOC concentration and using both field spectroradiometers and laboratory fixed spectrometers. To address this gap, we scanned 143 lowSOC-content soil samples using three spectrometers (ASD FieldSpec 3, ASD FieldSpec 4, and FOSS XDS) and four setup features - FOSS, contact probe (CP), dark-box (DB), and open laboratory (LAB) - at three laboratories. The application of an internal soil standard (ISS) to align one laboratory spectrum with another for spectral correction and spectral merging of various SSLs was examined. SOC models were developed using i) data from each single spectrometer - single laboratory separately and ii) merged data from multiple spectrometers - different laboratories, applying the $1^{\text {st }}$ derivatives of spectra and random forest (RF) regression. The results indicate that the spectral shape and position obtained from all spectrometers and setups did not show any noticeable differences, though spectra based on FOSS setup, particularly on low-SOC samples, demonstrated the highest absolute derivative values regardless of ISS application. The derivative ISS-corrected spectra showed less variation among different spectrometers compared to their uncorrected reflectance spectra. All single spectrometer models predicted SOC reasonably well. However, the spectra acquired by the FOSS setup predicted SOC more accurately $\left(\mathrm{R}^{2}=0.77\right.$ and $\mathrm{SD}=0.04)$ than the spectra acquired by the other setups. The models derived from merged uncorrected reflectance spectra yielded poor results $\left(\mathrm{R}^{2}=0.48\right.$ and $\left.\mathrm{SD}=0.10\right)$; nevertheless, assessment of SOC using the $1^{\text {st }}$ derivative ISS-corrected merged SSLs considerably improved the prediction accuracy $\left(\mathrm{R}^{2}=0.70\right.$ and $\left.\mathrm{SD}=0.06\right)$. Hence, the derivative spectra coupled with the ISS correction improved the accuracy of SOC prediction models obtained from merged soil spectra collected in different environments using different instruments. We therefore recommend application of the ISS spectral alignment method linked to the $1^{\text {st }}$ derivative approach to enhance the compilation of SSLs at the regional and global scales for SOC assessment.
\end{abstract}

Keywords: soil organic carbon, VIS-NIR-SWIR spectroscopy, instrument setup, scanning environment, soil spectral library. 


\section{Introduction}

49 Soil organic carbon (SOC) plays a fundamental role as an ecosystem indicator and a key parameter

50 of soil quality (Kibblewhite et al., 2008) and soil security (McBratney et al., 2014). Assessment of

51 SOC content has thus drawn considerable attention, in particular for agricultural soils with low

52 concentrations of SOC, where SOC levels risk reaching critical thresholds of, e.g., aggregate

53 stability (Le Bissonnais et al., 1996). As SOC significantly affects the shape and nature of soil

54 reflectance spectra (Gomez et al., 2008) and has strong spectral features (Ben-Dor et al., 1999; Liu

55 et al., 2019), it can be quantified quickly and affordably using visible-near infrared-shortwave

56 infrared (VIS-NIR-SWIR) spectroscopy (Ben-Dor and Banin, 1995; Viscarra Rossel et al., 2019;

57 Gholizadeh et al., 2020).

58 A major drawback in VIS-NIR-SWIR spectroscopy is that soil spectral information is sensitive to

59 scanning conditions (Ben-Dor et al., 2015), which profoundly influence measured reflectance

60 spectra and the derived models (Ge et al., 2011). Spectra collected in one specific laboratory are,

61 to some extent, influenced by the protocols used, e.g., sampling techniques, sample preparation,

62 temperature, and relative humidity (Pimstein et al., 2011; Chabrillat et al., 2019) or even by

63 scanning habits of different operators (Ge et al., 2011). Here, the term scanning environment

64 conditions indicates the abovementioned factors. Spectral performance and model accuracy may

65 vary significantly among different types of spectrometers or even among models from the same

66 manufacturer (Pimstein et al., 2011) due to equipment specification and setup, measurement

67 repeatability, geometry during spectral acquisition, calibration and performance of sensors,

68 efficiency of optical fibers, illumination source, and viewing angles of measurements (Ge et al.,

69 2011). We use the term instrument conditions to collectively refer to these factors.

70 Consequently, choosing the appropriate instrument and environment conditions is crucial for

71 effective soil spectra investigation; it is equally important to analyze, evaluate, and interpret the 
outputs. Different spectrometers such as ASD FieldSpec (Ben-Dor and Banin, 1995; Gholizadeh

73 et al., 2018b), PSR+ Spectral Evolution (Tsakiridis et al., 2017; Tzios et al., 2019), and FOSS XDS

74 (Genot et al., 2011; Stevens et al., 2013; Castaldi et al., 2018) were globally used to develop SOC

75 prediction models. Apart from the instrument, various analytical methods are used, which can also

76 significantly influence the prediction performance of the spectroscopic models and hence hinder

77 the exchange of SOC data between laboratories. If soil spectral libraries (SSLs), consisting of

78 thousands of soil samples, need to be merged, managing the large variation resulting from different

79 scanning environments and instrument conditions remains a challenging task. Given this issue,

80 other communities (e.g., food and fodder quality control) have developed a set of standard samples

81 to enable the exchange of data and models between scanning environments and instruments (Shenk

82 et al., 2001; Fearn, 2001; Ge et al., 2011). In the soil spectroscopy community, this issue has

83 recently been raised and only a few authors have started developing standards and protocols

84 including proposals for fixed geometry and spectral preprocessing (e.g. Ben-Dor et al., 2015);

85 however, it is still a matter of intense debate.

86 Knadel et al. (2013) used three VIS-NIR-SWIR spectrometers with varying resolutions and

87 spectral ranges under uniform laboratory conditions for SOC assessment to compare the performance of each single instrument, albeit without transferring the prediction models from one instrument to another. Ge et al. (2011) compared different VIS-NIR-SWIR spectrometers under different conditions for predicting SOC in the same population of soil samples with the same treatment (sampling technique, sample preparation, and sample roughness) applying the same 92 analysis technique (preprocessing, calibration, and validation). They used three calibration transfer 93 methods (slope and bias correction, direct standardization, and piecewise direct standardization) to 94 transfer calibration models from one instrument to another. Pimstein et al. (2011) minimized the 
protocol and the internal soil standard (ISS) concept. This concept is a simple realignment

97 procedure adopting an agreed-upon reference material that can be used to align one spectrum 98 (under any condition and setup) to another. It offers the possibility to align spectra from different 99 origins and merge them in a single SSL. Ben-Dor et al. (2015) introduced two sand dune samples 100 from the southwest coast of Australia as ISS samples, named from their location of origin as Lucky 101 Bay (LB) and Wylie Bay (WB). They were examined by various users (Ben-Dor et al., 2015; 102 Romero et al., 2018; Chabrillat et al., 2019; Crucil et al., 2019) to check their robustness for 103 alignment of the spectra obtained from different instruments. Generally, the LB (99\% quartz) 104 provided better results. Nevertheless, its capability to improve SOC prediction has only been tested 105 by Crucil et al. (2019), who trained SOC models using soil spectra acquired in laboratory 106 conditions for outdoor spectra with the alignment by the LB internal soil standard.

107 Given the importance of SOC assessment for agricultural soils with low SOC content, as well as 108 the effects of scanning environment and instrument conditions on the final performance of soil 109 prediction models, the current study was conducted under four different setups at three different laboratories: the Czech University of Life Sciences Prague (CZU), the German Research Center 111 for Geosciences (GFZ), and the University Catholique de Louvain (UCLouvain) with the aims of 112 i) comparing the low-SOC-content soil spectra measured with different spectrometers under 113 different scanning environment conditions, ii) examining the potential of three VIS-NIR-SWIR 114 spectrometers (ASD FieldSpec 3, ASD FieldSpec 4, and FOSS XDS) under four different setups 115 and different three laboratories to develop SOC models for agricultural soils of the Czech Republic, 116 and iii) assessing the potential of the ISS approach (LB sample) linked with common spectral 117 preprocessing to improve the predictability of SOC models derived from merged SSLs. 
118 The results contribute to implementing a rapid and low-cost procedure for SOC analysis, 119 particularly in agricultural soils with low SOC content, choosing a mature technique and a 120 satisfactory measurement protocol with appropriate performance.

\section{Materials and methods}

123 2.1. Study sites

124 Three typical agricultural sites with low SOC content from different parts of the Czech Republic

125 were chosen (Figure 1): Vidim $\left(50^{\circ} 460^{\prime} \mathrm{N}, 14^{\circ} 510^{\prime} \mathrm{E}\right.$, area 7 ha, altitude $315-323 \mathrm{~m}$ above sea level 126 (a.s.1.), average annual temperature $7-8^{\circ} \mathrm{C}$, average precipitation 550-650 mm), Sedlčany $\left(46^{\circ} 680^{\prime}\right.$ $127 \mathrm{~N}, 14^{\circ} 420^{\prime} \mathrm{E}$, area 5 ha, altitude $375-387 \mathrm{~m}$ a.s.1., average annual temperature $7-8{ }^{\circ} \mathrm{C}$, average 128 precipitation $550-650 \mathrm{~mm}$ ), and Brumovice $\left(48^{\circ} 960^{\prime} \mathrm{N}, 16^{\circ} 880^{\prime} \mathrm{E}\right.$, area 4 ha, altitude $187-227 \mathrm{~m}$ 129 a.s.l., average annual temperature $9-10{ }^{\circ} \mathrm{C}$, average precipitation $550-650 \mathrm{~mm}$ ).

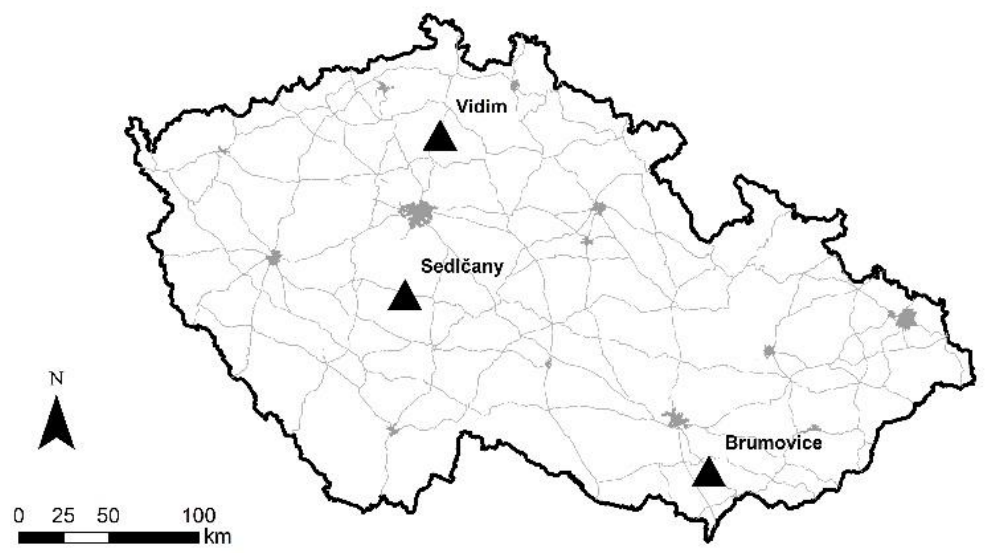

Figure 1. The Czech Republic and location of the study sites in the country.

132 According to the World reference base for soil resources (WRB, 2014), the original soil type in 133 Brumovice was a Haplic Chernozem on loess that transformed over time to a Regosol (steep parts) 134 and Colluvial soil (foot slope and the tributary valley) due to erosion and sedimentation. For Vidim, 
135 the original soil type was a Haplic Luvisol on loess, and for Sedlčany a Haplic Cambisol on gneiss.

136 A similar process of soil degradation resulting in a change of soil type can be detected at these sites 137 as well.

\subsection{Soil sampling and measurements}

140 We collected 143 soil samples from the topsoil $(0-20 \mathrm{~cm})$ within regular grids covering the sites

141 (Figure 2). Regarding field size, soil conditions, and selected sampling and analyzing algorithms

142 (Kuang and Mouazen, 2012; Ramirez-Lopez et al., 2014), the sample size had adequate coverage 143 of the predictor space and it was representative of the population to which the models were applied.

144 The position of each sampling point was recorded using a GeoXM (Trimble Inc., Sunnyvale, CA, 145 USA) receiver with accuracy of $1 \mathrm{~m}$.

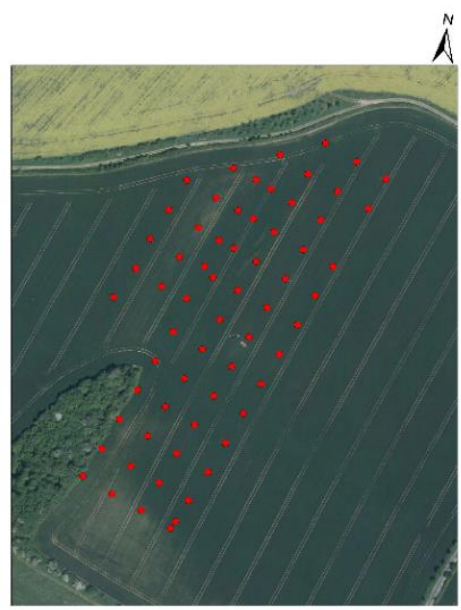

- Sampling points

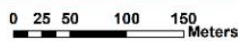

Vidim

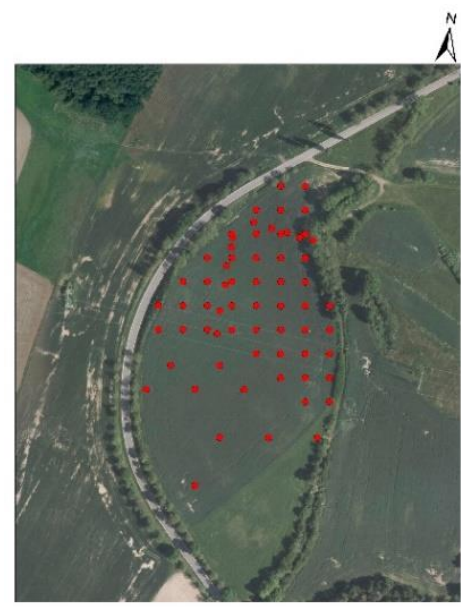

- Sampling points $\quad$\begin{tabular}{lllll}
0 & 25 & 50 & 100 & 150 \\
\hline
\end{tabular}

Sedlčany
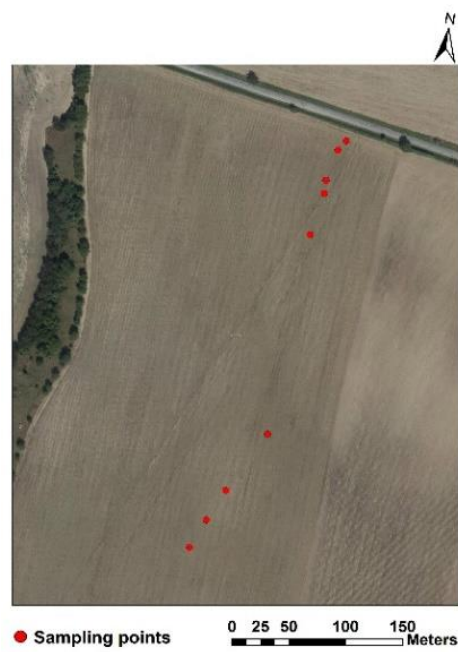

Brumovice

Figure 2. Location of sampling points.

The soil samples were air-dried, sieved to $\leq 2 \mathrm{~mm}$, and thoroughly mixed before analysis (ISO11464:2006). SOC was measured as total oxidized carbon (Table 1) using wet oxidation (ISO 14235:1998). 


\begin{tabular}{cc}
\hline Parameter & SOC $(\%)$ \\
\hline Mean & 1.28 \\
Median & 1.17 \\
Minimum & 0.62 \\
Maximum & 2.74 \\
SD & 0.2 \\
CV & 16 \\
\hline SD: standard deviation; CV: coefficient of variation
\end{tabular}

\subsection{VIS-NIR-SWIR spectroscopic measurements}

Table 2. Instruments specifications

\begin{tabular}{cccc}
\hline Spectrometer & Wavelength range & Spectral resolution & Spectral interval \\
\hline ASD FieldSpec 3 Pro FR & $350-2500 \mathrm{~nm}$ & $3 \mathrm{~nm} @ 350-1050 \mathrm{~nm}$ & $1.4 \mathrm{~nm} @ 350-1000 \mathrm{~nm}$ \\
& & $10 \mathrm{~nm} @ 1051-2500 \mathrm{~nm}$ & $2 \mathrm{~nm} @ 1001-2500 \mathrm{~nm}$ \\
ASD FieldSpec 4 Pro FR & $350-2500 \mathrm{~nm}$ & $3 \mathrm{~nm} @ 350-1050 \mathrm{~nm}$ & $1.4 \mathrm{~nm} @ 350-1000 \mathrm{~nm}$ \\
& & $10 \mathrm{~nm} @ 1051-2500 \mathrm{~nm}$ & $2 \mathrm{~nm} @ 1001-2500 \mathrm{~nm}$ \\
FOSS XDS Rapid Content Analyzer & $400-2498 \mathrm{~nm}$ & $0.5 \mathrm{~nm}$ & $2 \mathrm{~nm}$ \\
\hline
\end{tabular}

All instruments were operated under the routine protocols and conditions of their respective labs; some external factors such as temperature $\left(20-23{ }^{\circ} \mathrm{C}\right)$ and relative humidity $(40-45 \%)$ were almost similar. The ASD FieldSpec spectrometers measure using a Si Photodiode and an InGaAs SWIR detector. Their spectral resolution is $3 \mathrm{~nm}$ for the $350-1050 \mathrm{~nm}$ region and $10 \mathrm{~nm}$ for the $1051-$ $2500 \mathrm{~nm}$ region. ASD FieldSpec 3 and ASD FieldSpec 4 provide a spectral sampling of $1.4 \mathrm{~nm}$ in the $350-1000 \mathrm{~nm}$ spectral range and $2 \mathrm{~nm}$ in the $1001-2500 \mathrm{~nm}$ spectral range, although data are 170 interpolated to $1 \mathrm{~nm}$ intervals. The FOSS XDS spectrometer measures the reflectance continuously 171 in the 400-1300 $\mathrm{nm}$ region with a Si detector and 1300-2498 nm with a Pbs detector, offering a 
172 spectral resolution of $0.5 \mathrm{~nm}$ (4200 wavelengths). Measurements using ASD FieldSpec 4 at the

173 GFZ were recorded under two different setups: the common setup and a dark-box (DB)

174 environment developed at the GFZ. The details of each measurement protocol are presented in the

175 following sections.

176

177 2.3.1. Measurements at the CZU

178 For the spectra measurements by ASD FieldSpec 3 at the CZU, a high-intensity contact probe (CP), 179 powered by a $6.5 \mathrm{~W}$ tungsten-quartz-halogen lamp, was chosen (Figure 3 ). Here, we introduce this 180 setup as CP.

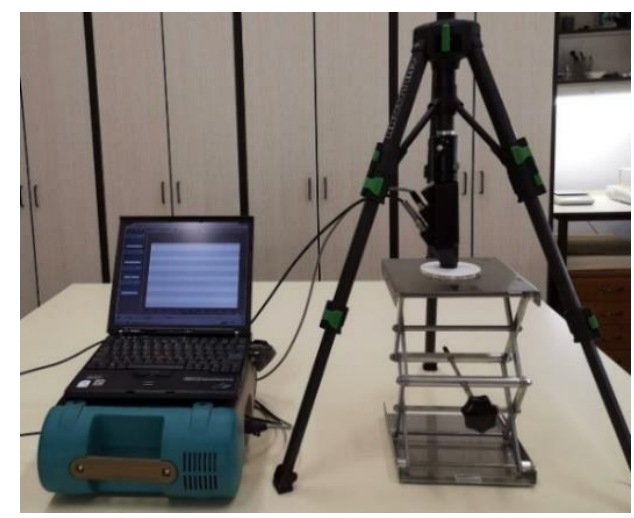

183 The instrument ran for 30 minutes to warm up the spectrometer and lamp. The soil samples were 184 placed in $5 \mathrm{~cm}$ diameter petri dishes and levelled with a stainless-steel blade (formed a $2 \mathrm{~cm}$ layer 185 of soil) to guarantee a flat surface flush with the top of the petri dish as a smooth soil surface ensures maximum light reflection and a high signal-to-noise ratio (SNR) (Mouazen et al., 2005).

187 The samples were lifted up to the $\mathrm{CP}$ assembly by a manual elevator and all spectral readings were 188 recorded in the center of the samples (three replications each). The spectrometer was calibrated 189 prior to the first and after every 10 measurements using a white Spectralon ${ }^{\mathrm{TM}}$ panel (Labsphere, 
191 (Ben-Dor et al., 2015).

\subsubsection{Measurements at the GFZ}

At the GFZ, two series of spectra measurements, both using ASD FieldSpec 4, were recorded under the common (LAB) and DB setups (Figure 4). Soil samples for spectra measurements were prepared in the same way as at the CZU (Section 2.3.1.).
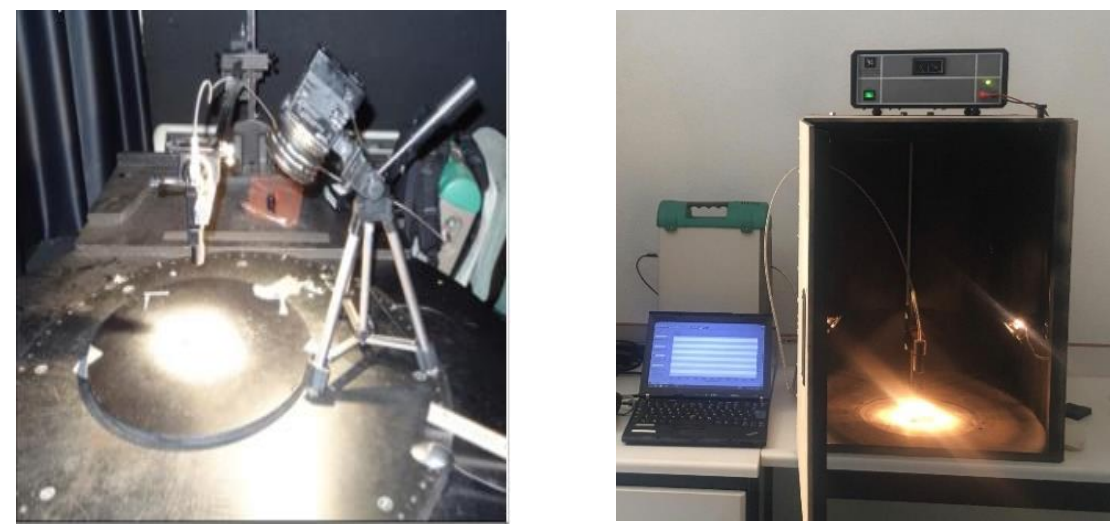

Figure 4. Measurement setups at the GFZ: LAB (left) and DB (right).

The spectral measurements under LAB setup were performed using an ASD Lamp Pro as the light source. Artificial light was installed at an $\sim 35^{\circ}$ zenith viewing angle and the instrument warmed up for 60 minutes before the actual scanning. The samples were located $\sim 7 \mathrm{~cm}$ in nadir below and in the middle of $8^{\circ}$ opening angle fore-optics and measured in the center of the samples (three replications each, rotating and steering the sample, flattening before each measurement) in a dark room to avoid interference from stray light. The spectrometer was optimized using a Spectralon ${ }^{\mathrm{TM}}$ white reference (Labsphere, North Sutton, NH, USA) at the beginning and end of each batch of 5 soil sample measurements. The LB sample was scanned after the Spectralon ${ }^{\mathrm{TM}}$ white reference at the beginning of each batch and before the next Spectralon ${ }^{\mathrm{TM}}$ measurement at the end of each batch. 
The DB was developed at the GFZ (Figure 5) following the concept reported by Ben-Dor et al.

210 (2015). The objective of the design was providing a measurement setup with fixed geometry for

211 lighting and variable samples. The distance of different objects to the sensor should be feasible and

212 samples can be rotated for taking multiple measurements. To exclude stray light from random light

213 sources, a wooden box was built; reflection the inside was further reduced by applying black paint

214 to the inside of the box. To reduce shading on uneven samples, two halogen bulbs were used for

215 illumination, both are pointing at $45^{\circ}$ downward at the sample. The selected bulbs had to be without

216 a front/diffusor window to avoid adding spectral transmission features. The lamps were powered

217 by a switching power supply and an uninterruptible power source (UPS) to avoid interference

218 artifacts due to voltage oscillations. The ASD fiber optics were mounted above the center of the

219 rotary table and different fore-optics can be attached to the mount.
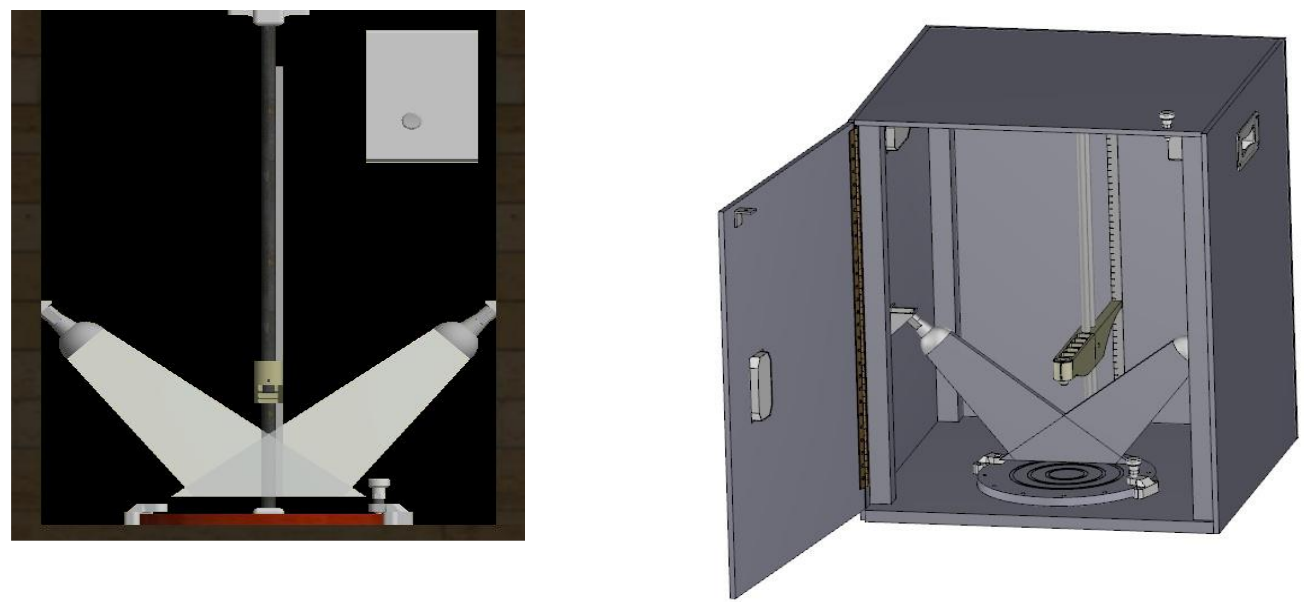

Figure 5. Photograph (left) and schematic (right) of the dark-box developed at the GFZ.

223 The spectra measurements under the DB setup were acquired with an $8^{\circ}$ fore-optic and a nadir 224 viewing sensor $\sim 7 \mathrm{~cm}$ above the target, producing a $\sim 1 \mathrm{~cm}$ ground instantaneous field of view 225 (GIFOV) in the middle of the sample. The same batch procedure was used as for the LAB setup 226 with three replication measurements (rotating and steering the sample, flattening before each 
227 measurement), optimization using a Spectralon ${ }^{\mathrm{TM}}$ white reference (Labsphere, North Sutton, NH,

228 USA), and acquisition of the LB sample measurements at the beginning and end of each batch of

2295 soil samples.

230

231 2.3.3. Measurements at REQUASUD commissioned by the UCLouvain

232 For soil spectra measurement using the FOSS XDS spectrometer (termed FOSS setup in this 233 paper), the samples were sent to Province of Liege Laboratories (member of the REQUASUD 234 network). According to the standard protocol of the REQUASUD laboratories for soil scanning 235 (Genot et al., 2011), two quarter cups were filled with soil material ( $25 \mathrm{~g})$ for each soil sample. 236 Thus, the soil samples were measured in duplicate, and for each subsample, an averaged 237 absorbance spectrum was obtained from 32 scans. The spectra averaged between the two 238 subsamples were recorded if the root mean square error (RMSE) between the two spectra of each 239 subsample was close enough based on a t-student test conducted with previously scanned samples; 240 otherwise, the average spectrum was discarded and the sample was scanned again using the same 241 procedure described above. The LB sand was scanned, following this procedure, every 20 soil 242 samples.

244 2.4. Spectral preprocessing, modelling, and evaluation

245 Before using the data in SOC models, spectral data obtained from all setups i) at each single 246 laboratory by each single spectrometer separately and ii) at different laboratories by multiple 247 spectrometers were preprocessed.

249 2.4.1. Single spectrometer - single laboratory 
250 For single laboratory modelling, the three replicates measurements were averaged. The noisy 251 portions between 350-399 $\mathrm{nm}$ and 2490-2500 nm (for ASD FieldSpec spectrometers) and 400$252447 \mathrm{~nm}$ (for FOSS XDS spectrometer) were removed and the reflectance spectra were subjected to 253 Savitzky-Golay smoothing with a second-order polynomial fit and 15 smoothing points to remove 254 the artificial noise caused by the spectrometer (Ren et al., 2009; Song et al., 2012). Then, the $1^{\text {st }}$ 255 derivative transformation was used as a spectral preprocessing algorithm. This is effective for 256 removing baseline offset and according to some researchers, produces the best results and higher 257 accuracy among the other techniques for developing soil spectral models (Duckworth, 2004; Ben258 Dor et al., 2008; Gholizadeh et al., 2015).

259 The datasets were divided into calibration ( $75 \%$ of samples) and validation ( $25 \%$ of samples) sets 260 using 100 times random stratified sampling (Gholizadeh et al., 2018a). For modelling, the ensemble 261 machine learning algorithm random forest (RF) regression was used (Breiman, 2001). RF works 262 by growing a group of regression trees based on binary recursive partitioning, where the feature 263 space is repeatedly split into regions involving observations with similar response values (Strobl et 264 al., 2009). It minimizes the risk of overfitting and using it almost no fine-tuning of parameters is 265 needed to produce good predictions (Diaz-Uriarte and Andres, 2006; Saberioon et al., 2018). To assess the model performance, standard model evaluation statistics: the coefficient of determination

$267\left(\mathrm{R}^{2}\right)$ between the observed and predicted values of SOC and standard deviation (SD) were used, 268 which were averaged for 100 repetitions in the validation set to quantify the validation accuracy every time. Fisher's least significant difference (LSD) test was then applied to evaluate the statistical differences to verify the existence of a significance difference in case of model accuracy

271 (Castaldi et al., 2016). 
275 For inter-laboratory modelling, the preprocessing procedure was conducted by applying the same 276 method as for each single laboratory (section 2.4.1.). However, to remove noise and non-linearity 277 effects, wavelengths were cut off to $448-2488 \mathrm{~nm}$. In addition, the ASD FieldSpec spectrometers 278 data were resampled to the $0.5 \mathrm{~nm}$ spectral resolution of the FOSS XDS spectrometer. In addition, 279 before transformation of the spectra to the $1^{\text {st }}$ derivative, the spectra were corrected by the ISS 280 standard. Following the suggestion of Pimstein et al. (2011) adopted by Ben-Dor et al. (2015), a

correction factor $(\mathrm{CF})$ was used in the ISS realignment procedure to align all setups. The reflectance value of each sample acquired from the different setups was corrected multiplying the $\mathrm{CF}$ with the original spectrum. The details of the $\mathrm{CF}$ and its implementation for spectral correction were previously described by Ben-Dor et al. (2015) and Chabrillat et al. (2019). Afterward, the model derived from the LB-corrected spectra of all laboratories and multiple spectrometers was developed. The random stratified sampling was used 100 times to provide the calibration set (75\% of samples) equally distributed in all laboratories (full range selection in each laboratory). Similar to the procedure used for single laboratories, RF was used for spectral modelling of SOC using the merged data of all laboratories. The final prediction accuracy was assessed using $\mathrm{R}^{2}$ and SD. These metrics were averaged for 100 repetitions in validation set equally distributed over all laboratories (full range selection in each laboratory).

\section{Results and discussion}

\subsection{Soil spectral features assessment}

The mean $1^{\text {st }}$ derivative LB-corrected spectra of all samples with different range of SOC contents (lower contents: $0.62-0.75 \%$ and higher contents: $2.02-2.74 \%$ ) scanned at three laboratories by three different spectrometers (under four different setups) are shown in Figure 6. They were also 

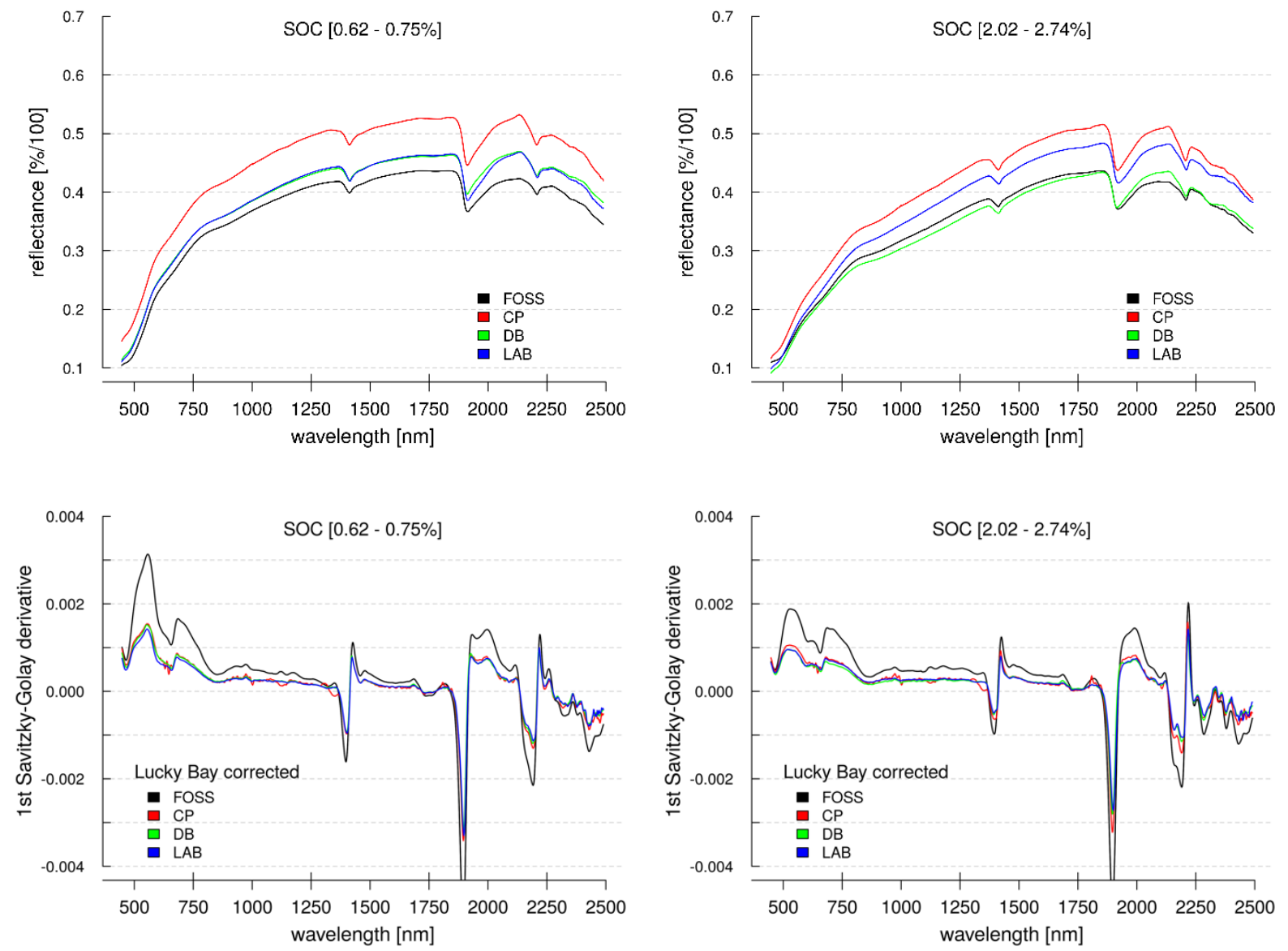

300 Figure 6. Mean spectra of samples with lower (left) and higher (right) range of SOC contents measured by the FOSS, CP, DB, and LAB setups at different laboratories: uncorrected reflectance spectra (top) and $1^{\text {st }}$ derivative LB-corrected spectra (bottom).

There were no significant changes in spectral features and the general shape of the curves remained constant under all scanning environments and instrument conditions within both uncorrected reflectance and $1^{\text {st }}$ derivative LB-corrected spectra categories and in both SOC ranges (Figure 6). Several absorption features, including features of Fe oxides in the VIS range (400-700 nm) were obvious in all spectra (particularly in derivative spectra) regardless of the SOC content ranges, instruments, and laboratories. The absorption features of $\mathrm{O}-\mathrm{H}$ bands at $1400 \mathrm{~nm}$ and $1900 \mathrm{~nm}$, and 
310 a doublet around $2200 \mathrm{~nm}$ related to the $\mathrm{O}-\mathrm{H}$ of hygroscopic water and clay minerals lattice (Ben-

311 Dor et al., 1999) were clearly observed. The curve and spectral features are similar to those 312 described by Ge et al. (2011) and Knadel et al. (2013) using multiple spectrometers, and Romero 313 et al. (2018) using ISS correction.

314 Figure 6 also highlights that despite the spectra's similar shape and spectral features position in 315 each group, the absorption intensities of spectra varied, mainly in the VIS range (as SOC is more 316 active in this region) of low-SOC samples depending on the spectrometer used. The differences 317 among spectra in absorption/reflectance intensities in the NIR and SWIR regions were smaller in 318 both reflectance and derivative spectra. The FOSS spectra, specifically for low-SOC samples, had 319 the highest absolute derivative values/the lowest reflectance values at almost all wavelengths 320 regardless of the preprocessing approach and LB application. This is probably due to the short 321 distance between the sample, light source, and the more controlled environment within the FOSS 322 setup. In a study by Romero et al. (2018), evaluating the effect of spectrometers' light source 323 distance to soil sample, the spectra derived from a CP setup with shorter target and illumination 324 distance showed higher values across the entire spectrum. They mentioned that the distance 325 between the light source and the target significantly influences the reflectance intensity of soil 326 samples. Hence, variation between sensors measuring the same soil samples may be caused by 327 noise during acquisition, integrity of the optical fibers, illumination variation, and the performance 328 of each detector in each spectral region (Pimstein et al., 2011). The same results were obtained by 329 Ge et al. (2011) and Chabrillat et al. (2019) measuring the same samples under various sensor 330 setups.

331 The uncorrected spectra (Figure 6, top) indicated that there were stronger variations among the 332 spectra, (particularly for low SOC contents) obtained from different spectrometers, setups, and 333 laboratories. These differences decreased after LB correction (Figure 6, bottom). This 
334 demonstrated the capability of the LB sample to minimize the existing systematic variations among

335 different scanning instruments and environment conditions. The methodology narrowed the

336 deviation of soil spectra (for the same samples) and reduced the differences between sensors and

337 laboratories, which was also reported by Gholizadeh et al. (2017) and Romero et al. (2018).

\subsection{Single spectrometer - single laboratory models for SOC prediction}

340 According to Ben-Dor et al. (2015), the ISS spectral realignment procedure improves SOC

341 prediction when models are calibrated using spectra from different sources. In order to show the

342 differences between different laboratories and measurement conditions and for development of the

343 SOC prediction models at each single laboratory (CZU, GFZ, and UCLouvain) using the spectrum

344 from each individual spectrometer setup (FOSS, CP, DB, and LAB), the $1^{\text {st }}$ derivative spectral 345 information without LB correction was used (Figure 7).

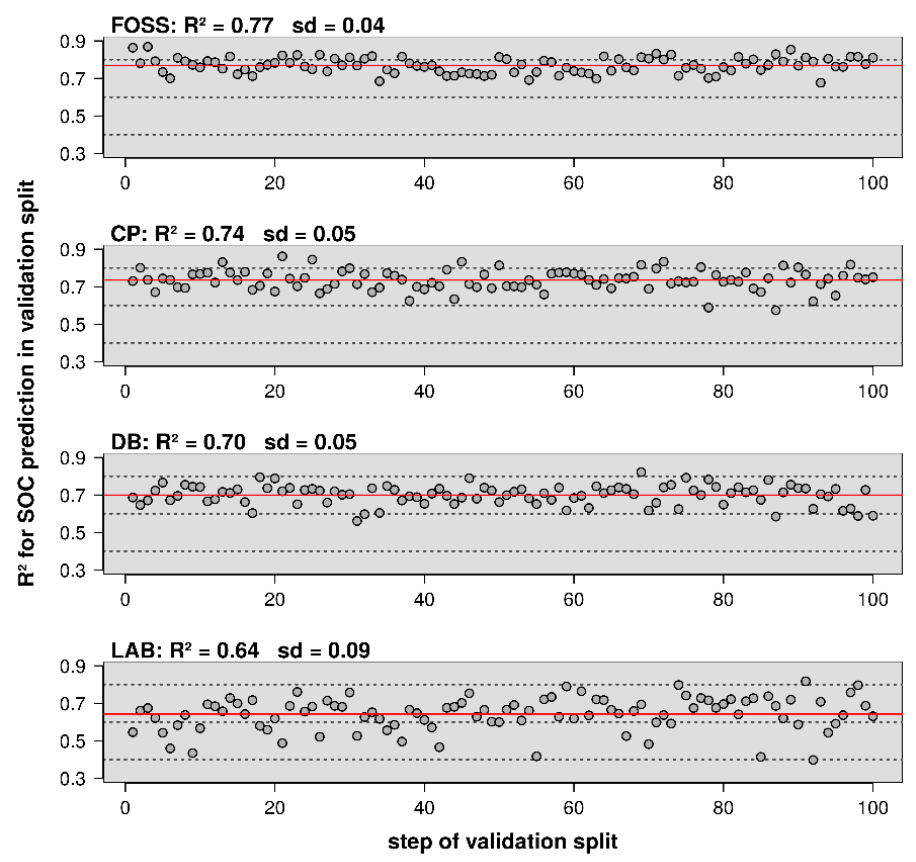

Figure 7. SOC prediction using single FOSS, CP, DB, and LAB spectrum obtained at UCLouvain, 349 
350 The model developed for SOC using the FOSS setup performed best with a $\mathrm{R}^{2}$ of 0.77 and SD of 3510.04 followed by the $\mathrm{CP}$ with $\mathrm{R}^{2}$ of 0.74 and $\mathrm{SD}$ of 0.05 . Both models are classified as having good 352 prediction accuracy (Chang et al., 2001). The LSD test carried out on the validation statistics of all 353 datasets also confirmed that the FOSS data provided significantly better estimation accuracy 354 compared to other instruments and setups (Table 3). The LAB setup, using ASD FieldSpec 4 Pro 355 FR in open laboratory conditions, had the lowest $\mathrm{R}^{2}(0.64)$ and the highest $\mathrm{SD}(0.09)$ in validation 356 performance, although according to Chang et al. (2001), these results are still fair $\left(0.5 \leq \mathrm{R}^{2} \leq 0.7\right)$.

357 The results of the SOC estimation produced by the DB configuration showed similar SD values of 3580.05 to the $\mathrm{CP}$-derived model. The model derived from spectra measured with a $\mathrm{CP}$ yielded a higher $359 \mathrm{R}^{2}$ of 0.74 . In addition, the LSD test detected a significant difference between DB and CP setups 360 for SOC assessment (Table 3).

Table 3. Least significant difference test

\begin{tabular}{ccc}
\hline Setup & $\mathbf{R}^{\mathbf{2}}$ & Group \\
\hline FOSS & 0.77 & $\mathrm{a}$ \\
CP & 0.74 & $\mathrm{~b}$ \\
DB & 0.70 & $\mathrm{c}$ \\
LAB & 0.64 & $\mathrm{~d}$ \\
\hline
\end{tabular}

364 Spectrometers are governed by the instrument's stability, SNR, configuration, illumination source, 365 detector performance, fore-optic and fiber optic characteristics, stray light contribution, and warm366 up time (Ben-Dor et al., 2015). Therefore, the variation in modelling accuracy can be expected. 367 These differences can also be considered as the expected errors in routine standards applied in the 368 laboratories and even operators' measurement habits (Genot et al., 2011; Pimstein et al., 2011). In 369 contrast, Knadel et al. (2013) and Romero et al. (2018) found no variation in clay modeling 370 performance between three different field spectrometers. The best performance using the FOSS 371 XDS can be explained by its particular advantages of a closed environment, fixed light sources, 
372 and stability of the probe measurements, which reduce the possibility of error. Regarding the CP

373 setup, acceptable results were obtained that can be attributed to the use of a mechanical stand for

374 holding the contact probe, decreasing the vibrations compared to a handheld setup (Pimstein et al.,

375 2011). The lower predictive power of the LAB setup is probably caused by stray light that can be

376 connected to the single light source and distance between object and light source (Ge et al., 2011).

377

\subsection{Multiple spectrometers - inter laboratory models for SOC prediction}

379 For inter-laboratory modelling, the datasets obtained from each spectrometer at each laboratory 380 were merged to develop a single SOC model. For calibration of the models, the LB correction was 381 first applied on the reflectance spectra. The spectra were then transformed to Savitzky-Golay $+1^{\text {st }}$ 382 derivative spectra. The model was compared with the models obtained from merged uncorrected 383 reflectance spectra as a reference (Figure 8).

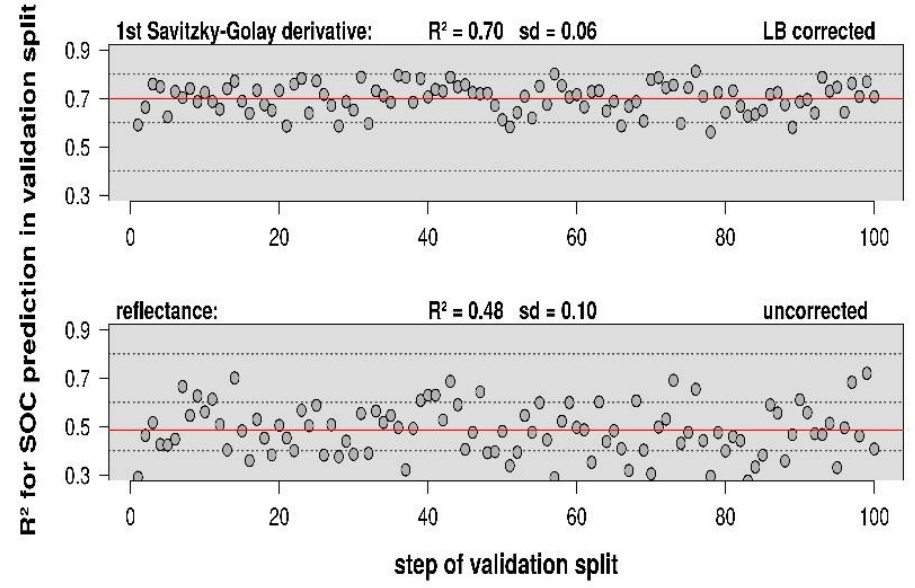

Figure 8. SOC prediction using FOSS-CP-DB-LAB merged spectra obtained at different laboratories with (top) and without LB correction (bottom). The red line indicates the averaged $\mathrm{R}^{2}$ for 100 validation splits $(25 \%)$.

The predicted SOC from the uncorrected reflectance spectra presented an obvious wider scattering with low accuracy and poor performance $\left(\mathrm{R}^{2}=0.48\right.$ and $\left.\mathrm{SD}=0.10\right) . \mathrm{R}^{2}$ was considerable higher 
391 value $\left(\mathrm{R}^{2}=0.70\right)$ and SD showed a lower value (0.06), when using derivative spectra coupled with 392 the LB correction in comparison with uncorrected reflectance spectra. The ISS correction (LB) 393 improved the prediction accuracy of SOC using reflectance spectra due to the capability of the LB 394 sample to align the spectra acquired in different laboratories and using multiple spectrometers 395 (Ben-Dor et al., 2015). Generally, the optimal case was combining the derivative spectra and LB 396 standard to increase inter-laboratory comparability. The suitability of this approach and derivative 397 spectra agrees with the results reported by Pimstein et al. (2011), who confirmed a better 398 performance of using the derivatives coupled with ISS correction compared to using raw 399 reflectance spectra.

400 The SOC models calibrated on the merged SSLs were slightly less accurate compared to most of 401 the single models that were calibrated on a single instrument in a single laboratory (Figures 7 and 402 8). This can be expected as, by combining datasets, we introduce more sources of variation as a 403 result of different scanning environments and instrument conditions (Ge et al., 2011). It can also 404 be explained that if a strict protocol (geometry, spectrometer, environmental condition, and users) 405 is used, even without correction, the prediction performance would be reliable as a result of the 406 smaller systematic errors by applying a precise protocol (Romero et al., 2018). Nonetheless, 407 merging SSLs using spectral derivatives and standards such as ISS correction (the LB sample in 408 this study) can lead to the development of a regional/global calibration model that extends the 409 applicability of the model at the cost of a small reduction in prediction accuracy. This is particularly 410 important considering the recent developments such as the European Land Use/Cover Area Survey 411 (LUCAS) SSL that could be spiked or merged with local SSLs from different research institutes 412 (Orgiazzi et al., 2018).

413 Notably, here, we dealt with three spectrometers, four setup features and three laboratories, as well 414 as the same soil sample set. However, for development of a large-scale SSL with diverse soil 
415 samples and scanning conditions, the promising role of the ISS approach linked to spectral 416 preprocessing techniques in providing acceptable prediction accuracies is more evident (Ben-Dor 417 et al., 2015; Chabrillat et al., 2019). In this case, a more significant improvement of the model 418 performance can be observed.

\section{Conclusions}

421 Based on the spectral analyses of 143 agricultural soil samples with a low SOC content, the spectral 422 shape and position obtained from different spectrometers and setups (FOSS, CP, DB, and LAB) 423 did not show any noticeable differences, but there was a variation in the absorption intensities of 424 spectra acquired by the spectrometers, particularly in the VIS range. The spectra based on the FOSS 425 setup yielded the highest absolute derivative values/the lowest reflectance values at almost all 426 wavelengths. The derivative spectra corrected with the LB standard indicated less variation among 427 different spectrometers' spectra compared to their uncorrected reflectance spectra. The results also 428 highlighted that despite the fair to good calibration accuracy of all single models, the derivative 429 spectra obtained from the FOSS XDS spectrometer, which scans under more stable and controlled 430 conditions, produced in a more reliable SOC prediction model compared to other spectrometers, 431 which was confirmed by the LSD test. When the reflectance spectra from multiple spectrometers 432 and laboratories were merged, the outputs were poor, although the $1^{\text {st }}$ derivative LB-corrected 433 spectra of soil mixed from different scanning environments and instrument conditions significantly 434 improved the model accuracy. In this case, the findings showed that the sets of derivative spectra 435 matched remarkably with the LB correction minimizing those variations enabling the merging data 436 process, which is an essential part of establishing any SSL. Nowadays, when users are sharing data 437 derived from diverse soil samples, different scanning environments, and various instruments for 438 building SSLs and developing regional/global models; the use of standards (e.g., the LB approach) 
439 for reducing the variation and better merging of soil spectral databases is recommended. This

440 allows direct comparison and use in quantitative models of spectra acquired in diverse conditions.

441 In this study, we focused only on SOC (mainly low contents) in one set of soil samples; however,

442 other soil properties and several soil datasets still need further investigation.

\section{Acknowledgement}

445 The authors like to thank the financial support of the Czech Science Foundation (project No. 1828126Y). The GFZ is acknowledged for the financial support of Asa Gholizadeh for 6 weeks 447 research stay at the GFZ in 2017 under a short-term guest scientist stay grant. The authors further 448 acknowledge the support of European Regional Development Fund, project Centre for the 449 investigation of synthesis and transformation of nutritional substances in the food chain in interaction with potentially harmful substances of anthropogenic origin: comprehensive assessment of soil contamination risks for the quality of agricultural products (project No. 452 CZ.02.1.01/0.0/0.0/16_019/0000845). Eyal Ben-Dor, Cindy Ong, and Ian Lau are greatly appreciated for recommendations on the dark-box development.

\section{References}

Ben-Dor, E., Banin, A., 1995. Near-infrared analysis as a rapid method to simultaneously evaluate several soil properties. Soil Sci. Soc. Am. J. 59, 364-372.

Ben-Dor, E., Heller, D., Chudnovsky, A., 2008. A novel method of classifying soil profiles in the field using optical means. Soil Sci. Soc. Am. J. 72, 1113-1123. In: Rencz, Andrew N. (Ed.), 3rd ed. Manual of Remote Sensing, pp. 111-188.

Ben-Dor, E., Ong, C., Lau, I.C., 2015. Reflectance measurements of soils in the laboratory: standards and protocols. Geoderma. 245-246, 112-124.

Breiman, L., 2001. Random forests. Mach. Learn. 45, 5-32.

Castaldi, F., Chabrillat, S., Chartin, C., Genot, V., Jones, A.R., van Wesemael, B., 2018. Estimation of soil organic carbon in arable soil in Belgium and Luxembourg with the LUCAS topsoil database. Eur. J. Soil Sci. 69, 592-603. 
Castaldi, F., Palombo, A., Santini, F., Pascucci, S., Pignatti, S., Casa, R., 2016. Evaluation of the potential of the current and forthcoming multispectral and hyperspectral imagers to estimate soil texture and organic carbon. Remote Sens. Environ. 179, 54-65.

Chabrillat, S., Gholizadeh, A., Neumann, C, Berger, D., Milewski, R., Ogen, Y., Ben-Dor, E., 2019. Preparing a soil spectral library using the Internal Soil Standard (ISS) method: Influence of extreme different humidity laboratory conditions. Geoderma. 355, 113855.

Chang, C., Laird, D., Mausbach, M.J., Hurburgh, C., 2001. Near-infrared reflectance spectroscopy - principal component analysis of soil properties. Soil Sci. Soc. Am. J. 65, 480-480.

Crucil, G., Castaldi, F., Aldana-Jague, E., van Wesemael, B., Macdonald, A., van Oost, K., 2019. Assessing the performance of UAS-compatible multispectral and hyperspectral sensors for soil organic carbon prediction. Sustainability. 11, 1889.

Diaz-Uriarte, R., Andres, S., 2006. Gene selection and classification of microarray data using random forest. BMC Bioinform.7, 3.

Duckworth, J., 2004. Mathematical data preprocessing. In: Roberts, C.A., Workman Jr.J., Reeves IIIJ.B. (Eds.), Near-infrared Spectroscopy in Agriculture. ASA-CSSA-SSSA, Madison, WI., USA, pp. 115-132.

Fearn, T., 2001. Standardisation and calibration transfer for near infrared instrument: a review. J. Near Infrared Spec. 9, 229-244.

Ge, Y., Morgan, C.L.S., Grunwald, S., Brown, D.J., Sarkhot, D.V., 2011. Comparison of soil reflectance spectra and calibration models obtained using multiple spectrometers. Geoderma. 161, 202-211.

Genot, V., Colinet, G., Bock, L., Vanvyve, D., Reusen, Y., Dardenne, P., 2011. Near infrared reflectance spectroscopy for estimating soil characteristics valuable in the diagnosis of soil fertility. J. Near Infrared Spec. 19, 117-138.

Gholizadeh, A., Boruvka, L., Vasat, R., Saberioon, M.M., Klement, A., Kratina, J., Tejnecky, V., Drabek, O., 2015. Estimation of potentially toxic elements contamination in anthropogenic soils on a brown coal mining dumpsite by reflectance spectroscopy: a case study. PLoS One. 10(2), e0117457.

Gholizadeh, A., Carmon, N., Klement, A., Ben-Dor, E., Boruvka, L., 2017. Agricultural soil spectral response and properties assessment: Effects of measurement protocol and data mining technique. Remote Sens. 9, 1078.

Gholizadeh, A., Saberioon, M., Carmon, N., Boruvka, L., Ben-Dor, E., 2018a. Examining the performance of PARACUDA-II data-mining engine versus selected techniques to model soil carbon from reflectance spectra. Remote Sens. 10, 1172.

Gholizadeh, A., Saberioon, M., Viscarra Rossel, R.A., Boruvka, L., Klement, A., 2020. Spectroscopic measurements and imaging of soil colour for field scale estimation of soil organic carbon. Geoderma. 357, 113972.

Gholizadeh, A., Zizala, D., Saberioon, M., Boruvka, L., 2018b. Soil organic carbon and texture retrieving and mapping using proximal, airborne and Sentinel-2 spectral imaging. Remote Sens. Environ. 218, 89-103.

Gomez, C., Viscarra Rossel, R.A., McBratney, A.B., 2008. Soil organic carbon prediction by hyperspectral remote sensing and field vis-NIR spectroscopy: An Australian case study. Geoderma. 146, 403-411.

Kibblewhite, M.G., Ritz, K., Swift, M.J., 2008. Soil health in agricultural systems. In Philosophical Trans. R. Soc. B: Biol. Sci. 363, 685-701. 
Knadel, M., Stenberg, B., Deng, F., Thomsen, A., Greve, M.H., 2013. Comparing predictive abilities of three visible-near infrared spectrophotometers for soil organic carbon and clay determination. J. Near Infrared Spec. 21, 67-80.

Kuang, B., Mouazen, A.M., 2012. Influence of the number of samples on prediction error of visible and near infrared spectroscopy of selected soil properties at the farm scale. Eur. J. Soil Sci. 63, 421-429.

Lal, R., 2016. Soil health and carbon management. Food Energy Secur. 5, 212-222.

Le Bissonnais, Y., 1996. Aggregate stability and assessment of soil crustability and erodibility: I. Theory and methodology. Eur. J. Soil Sci. 47, 425-437.

Liu, S., Shen, H., Chen, S., Zhao, X., Biswas, A., Jia, X., Shi, Z., Fang, J., 2019. Estimating forest soil organic carbon content using vis-NIR spectroscopy: Implications for large-scale soil carbon spectroscopic assessment. Geoderma. 348, 37-44.

Mouazen, A.M., de Baerdemaeker, J., Ramon, H., 2005. Towards development of on-line soil moisture content sensor using a fibre-type NIR spectrophotometer. Soil Tillage Res. 80, 171183.

Orgiazzi, A., Ballabio, C., Panagos, P., Jones, A., Fernandez-Ugalde, O., 2018. LUCAS Soil, the largest expandable soil dataset for Europe: a review. Eur. J. Soil Sci. 69, 140-153.

Pimstein, A., Notesko, G., Ben-Dor, E., 2011. Performance of three identical spectrometers in retrieving soil reflectance under laboratory conditions. Soil Sci. Soc. Am. J. 75, 110-174.

Ramirez-Lopez, L., Schmidt, K., Behrens, T., van Wesemael, B., Dematte, J.A.M., Scholten, T., 2014. Sampling optimal calibration sets in soil infrared spectroscopy. Geoderma. 226-227, $140-150$.

Ren, H.Y., Zhuang, D.F., Singh, A.N., Pan, J.J., Qid, D.S., Shi, R.H., 2009. Estimation of As and $\mathrm{Cu}$ contamination in agricultural soils around a mining area by reflectance spectroscopy: a case study. Pedosphere. 19, 719-726.

Romero, D.J., Ben-Dor, E., Dematte, J.A.M., e Souza, A.B., Vicente, L.E., Tavares, T.R., Martello, M., Strabeli, T.F., da Silva Barros, P.P., Fiorio, P.R., Gallo, B.C., Sato, M.V., Eitelwein, M.T., 2018. Internal soil standard method for the Brazilian soil spectral library: Performance and proximate analysis. Geoderma. 312, 95-103.

Saberioon, M.M., Cisar, P., Labbe, L., Soucek, P., Pelissier, P., Kerneis, T., 2018. Comparative performance analysis of support vector machine, random forest, logistic regression and $\mathrm{k}$ nearest neighbours in Rainbow Trout (Oncorhynchus Mykiss) classification using image-based features. Sensors. 18, 1027.

Shenk, J.S., Workman Jr., J.J., Westerhaus, M.O., 2001. Application of NIR spectroscopy to agricultural products. In: Burns, D.A., Ciurczak, E.W. (Eds.), Handbook of Near-Infrared Analysis. Marcel Dekker Inc., New York, NY, pp. 419-474.

Song, Y., Li, F., Yang, Z., Ayoko, G.A., Frost, R.L., Ji, J., 2012. Diffuse reflectance spectroscopy for monitoring potentially toxic elements in the agricultural soils of Changjiang river delta, China. Appl. Clay Sci. 64, 75-83.

Stevens, A., Nocita, M., Toth, G., Montanarella, L., van Wesemael, B., 2013. Prediction of soil organic carbon at the European scale by visible and near infrared reflectance spectroscopy. PLoS One 8 (6), e66409.

Strobl, C., Malley, J., Tutz, G. 2009. An introduction to recursive partitioning: Rationale, application, and characteristics of classification and regression trees, bagging, and random forests. Psychol. Methods. 14, 323.

Tsakiridis, N.L., Tziolas, N., DImitrakos, A., Galanis, G., Ntonou, E., Tsirika, A., Terzopoulou, E., Kalopesa, E., Zalidis, G.C., 2017. Predicting soil properties for sustainable agriculture using 
560 vis-NIR spectroscopy - a case study in northern Greece. In: Proceedings of SPIE - The 561 International Society for Optical Engineering.

562 Tziolas, N., Tsakiridis, N.L., Ben-Dor, E., Theocharis, J., Zalidis, G.C., 2019. A memory-based 563 learning approach utilizing combined spectral sources and geographical proximity for improved 564 VIS-NIR-SWIR soil properties estimation. Geoderma. 340,11-24.

565 Viscarra Rossel, R.A., Lee, J., Behrens, T., Luo, Z., Baldock, J., \& Richards, A., 2019. Continental566 scale soil carbon composition and vulnerability modulated by regional environmental controls. $567 \quad$ Nat. Geosci. 12, 547-552.

568 WRB, 2014. World Reference Base for Soil Resources. Technical Report FAO Rome, Italy. 569 Available online: http://www.fao.org/3/ai3794e.pdf (accessed on 1 October 2015). 